\title{
A Novel Weighted Complex Evidence Combination with Its Application in Multisource Information Fusion
}

\section{Huaping He}

Southwest University

Liting He

Southwest University

Fuyuan Xiao ( $\nabla$ doctorxiaofy@hotmail.com)

Southwest University https://orcid.org/0000-0002-4304-7189

\section{Research Article}

Keywords: Complex evidence theory, Complex mass function, Weighted complex evidence distance, Multisource information fusion, Decision making, Medical diagnosis

Posted Date: October 27th, 2021

DOI: https://doi.org/10.21203/rs.3.rs-672333/v1

License: (a) (1) This work is licensed under a Creative Commons Attribution 4.0 International License. Read Full License 


\title{
A Novel Weighted Complex Evidence Combination with Its Application in Multisource Information Fusion
}

\author{
Huaping $\mathrm{He}^{\mathrm{a}}$, Liting $\mathrm{He}^{\mathrm{a}}$, Fuyuan Xiao ${ }^{\mathrm{a}, *}$ \\ ${ }^{a}$ School of Computer and Information Science, Southwest University, No.2 Road, BeiBei \\ District, Chongqing 400715, China
}

\begin{abstract}
With the development of evidence theory, classical Dempster-Shafer evidence theory has been extended to complex plane, called complex evidence theory. However, counterintuitive result may occurs in the case when fusing conflicting complex evidences. To address this problem, a new multisource information fusion method is proposed by means of complex evidential distance function. This proposed method can reduce the impact of abnormal complex evidence on the fusion results to better support decision. A numerical example and an application of medical diagnosis verify the feasibility and effectiveness of the proposed fusion method.

Keywords: Complex evidence theory, Complex mass function, Weighted complex evidence distance, Multisource information fusion, Decision making, Medical diagnosis
\end{abstract}

\section{Introduction}

In real-numbers world, uncertainty [1 3] is an inevitable problem in decision making [4, 5], especially in the field of information fusion [6]. Plenty

\footnotetext{
${ }^{*}$ Corresponding author: Fuyuan Xiao (e-mail: doctorxiaofy@hotmail.com).
} 
of methodologies had been developed, such as $D$-number [7, 8, $Z$-number [9, 10], ordered weighted averaging method [11, entropy [12 14], Dengentropy-based [15] and classical Dempster-Shafer's evidence theory (D-S evidence theory) [16-19]. Those relevant theories have been widely used in uncertainty [20], such as fuzzy set processing [21 24], soft set processing [25 27], fault diagnosis [28, 29], data fusion [30 32], group design [33, 34], industrial system [35], model building [36] 39] and Elbow method [40].

The classical D-S evidence theory as a more effective way to deal with uncertainty than Bayes probability theory [41]. The fusion results provided by the classical D-S evidence theory are fault-tolerant that support better performance in decision-making [42 45] and risk analysis [46]. The classical D-S evidence theory defines basic belief assignments (BPA) [47] and offers a fusion formula, Dempster's conation rule (DCR) for multi-group BPA fusion. Two functions, belief function [48] and plausibility function [49], come up sequentially, which assist to measurement [50, 51] modling [52] and decision making [53 55]. In the process of inheriting the evidence theory [56, 57], it is clarified that the DCR has inconsistent with the fact. Therefore, the amount of theories use evidence distance to modify fusion formula to improve fusion results [58, 59].

With the development of evidence theory, the classical D-S evidence theory has been extended to complex plane. In particular, the proposed complex mass function [60 63] and the generalized Dempster's rule of combination (GDRC) provide a new solution for the application of uncertainty problems [64, 65] in complex plane, hence, the complex evidence theory has the potential to be applied for complex fuzzy set processing [66 68]. Furthermore, 
when the phase or periodicity of uncertain data varies, complex evidence theory is able to decrease the influence of abnormal evidence in the process of processing. However, when fusing conflicting evidence evidence, counterintuitive results may arise.

Therefore, in this paper, a novel multi-source information fusion for complex basic belief assignments (CBBAs) [69] was proposed. The proposed method inspired by Deng's approach in the credibility of each evidence into account, provides different weights to the fusion according to different credibility additionally. In the case of highly inconsistent evidence and lack of identification framework, this method reduces the interference of abnormal CBBA. A numerical example is provided to illustrate the proposed method occupied more feasibility and rationality than the previous methods. Finally, fusion is effectively applied to practical problems.

The main contributions of this article are listed as follows:

1) The proposed method is an extension of GDRC. Compared with GDRC, the proposed method has an extraordinary performance in decision making. The proposed method can better eliminate the negative effects of conflicting data.

2) The proposed method satisfied the axioms of the distance and obtains the properties of nondegeneracy, boundedness and negativity.

3) This article provides an identification decision calculation example based on the proposed method. In addition, the availability of the proposed method verified by a numerical example and an application of the proposed method.

The general conclusion of this paper is as follows. Section 2 describes 
the preparatory work that will be used. In Section 3, the definition and specific implementation method of the proposed method will be discussed. A numerical example and an application of medical diagnosis will be mentioned in Section 4 and Section 5 sequentially. Finally, Section 6 will draw the conclusion of this articale.

\section{Preliminaries}

In this part, we will briefly introduce some existing methods to be used in this paper.

\subsection{Complex evidence theory}

The classical D-S evidence theory is extended to the complex field which made the complex generalized belief function [70, 171] a great process. Complex evidence theory in the field of complex plane can express the ability of data to express the fluctuation of specific stages in the process of execution. In the process of dealing with uncertain and imprecise data, complex evidence theory is able to reduce the impact of abnormal complex evidence in the process of data molding and processing.

Definition 1. A set of mutually exclusive and collective nonempty events, which recorded as $\Omega=\left\{E_{1}, E_{2}, E_{3}, \ldots, E_{n-1}, E_{n}\right\}$. And it is called the frame of disturbance. Then the power set of $\Omega, 2^{\Omega}$ we defined as:

$$
2^{\Omega}=\left\{\varnothing,\left\{E_{1}\right\},\left\{E_{2}\right\}, \ldots,\left\{E_{i}\right\},\left\{E_{1}, E_{2}\right\}, \ldots,\left\{E_{1}, E_{2} \ldots E_{n-1}, E_{n}\right\}, \Omega\right\},
$$

where $\varnothing$ means an empty set. And the $C B B A$ is expressed as follows:

$$
\begin{gathered}
\mathbb{M}(\varnothing)=0 \\
\mathbb{M}(A)=m(A) e^{i \theta(A)}=x+y i, A \in 2^{\Omega},
\end{gathered}
$$




$$
\sum_{A \subseteq P(\Omega)} \mathbb{M}(A)=1,
$$

where

$$
i=\sqrt{-1}, m(A) \in[0,1], \Omega(A) \in[-\pi, \pi] .
$$

CBBA can also be called complex mass function (CMF), which can reflect the degree of trust we have in different events. If there are two sets of CBBAs, the GDR is needed to make a final decision.

Definition 2. If there are two sets of $C B B A$ s evidence $\mathbb{M}_{1}$ and $\mathbb{M}_{2}$. The GDR definition of these two evidences is $\mathbb{M}=\mathbb{M}_{1} \oplus \mathbb{M}_{2}$. This process is as follows:

$$
\begin{aligned}
\mathbb{M}(A) & =\frac{\sum_{B \cap C=A} \mathbb{M}_{1}(B) \mathbb{M}_{2}(C)}{\mathbb{K}}, \\
\mathbb{K} & =\sum_{B \cap C=\varnothing} \mathbb{M}_{1}(B) \mathbb{M}_{2}(C) .
\end{aligned}
$$

According to GDR we can get a new CBBA after two CBBAs $\mathbb{M}_{1}$ and $\mathbb{M}_{2}$ fusion, which is convenient to synthesize the two evidences for further decisionmaking.

\subsection{Complex evidence distance (CED)}

In evidence theory, evidence distance measurement also plays an important role, which leads to many scholars' researches. One of the most famous methods are Jousselme's distance [72 74]. But it based on the real number range. In the field of complex plane, Xiao proposed a complex evidence distance [75]. This distance is defined on the complex basic belief assignments.

Definition 3. Let $\mathbb{M}_{1}$ and $\mathbb{M}_{2}$ be two independent CBBAs in the frame of discernment $\Omega$. CED, the distance of $\mathbb{M}_{1}$ and $\mathbb{M}_{2}$ is defined as:

$$
d_{C B B A}\left(\mathbb{M}_{1}, \mathbb{M}_{2}\right)=\sqrt{\frac{\left\|\overrightarrow{\mathbb{M}}_{1}\right\|^{2}+\left\|\overrightarrow{\mathbb{M}}_{2}\right\|^{2}-2\left|\left\langle\overrightarrow{\mathbb{M}}_{1}, \overrightarrow{\mathbb{M}}_{2}\right\rangle\right|}{2}},
$$


where

$$
\left|\left\langle\overrightarrow{\mathbb{M}}_{1}, \overrightarrow{\mathbb{M}}_{2}\right\rangle\right|=\left|\sum_{i=1}^{2^{n}} \sum_{j=1}^{2^{n}} \mathbb{M}_{1}\left(A_{i}\right) \overline{\mathbb{M}}_{2}\left(A_{j}\right) \frac{\left|A_{i} \cap A_{j}\right|}{\left|A_{i} \cup A_{j}\right|}\right|,
$$

and the $\left\|\overrightarrow{\mathbb{M}}_{n}\right\|^{2}$ can be regarded as:

$$
\left\|\overrightarrow{\mathbb{M}}_{n}\right\|^{2}=\left|\left\langle\overrightarrow{\mathbb{M}}_{n}, \overrightarrow{\mathbb{M}}_{n}\right\rangle\right|
$$

The distance between $\mathrm{CBBAs} \mathbb{M}_{1}$ and $\mathbb{M}_{2}$ can be calculated by Eq. (7, 9 ). The distance between groups of CBBAs can be written in a two dimensional matrix. The elements in the matrix are the distance between each CBBAs.

\section{A new multisource information fusion for CBBAs}

In the process of complex evidence fusion, the conflicting evidence has a great influence on the result of GDRC. So our method will assign a weight value according to the difference between different complex evidences. In this section, the weighted average complex combination rule is proposed. This proposed method using Xiao's distance to calculate the distance of several CBBAs. Then using it to calculate the modified average complex evidence (MACE). Then the final result is fused by MACE.

Here are the steps of the proposed method:

Step 1: The distance between CBBAs is calculated based on Eq. (7) 9). Then construct a complex distance matrix $\mathcal{D}=\left(d\left(\mathbb{M}_{i}, \mathbb{M}_{j}\right)\right)_{n \times n}$ as follows:

$$
\mathcal{D}=\left[\begin{array}{ccccc}
0 & d\left(\mathbb{M}_{1}, \mathbb{M}_{2}\right) & \cdots & d\left(\mathbb{M}_{1}, \mathbb{M}_{n-1}\right) & d\left(\mathbb{M}_{1}, \mathbb{M}_{n}\right) \\
d\left(\mathbb{M}_{2}, \mathbb{M}_{1}\right) & 0 & \cdots & d\left(\mathbb{M}_{2}, \mathbb{M}_{n-1}\right) & d\left(\mathbb{M}_{2}, \mathbb{M}_{n}\right) \\
\vdots & \vdots & \ddots & \vdots & \vdots \\
d\left(\mathbb{M}_{n-1}, \mathbb{M}_{1}\right) & d\left(\mathbb{M}_{n-1}, \mathbb{M}_{2}\right) & \cdots & 0 & d\left(\mathbb{M}_{n-1}, \mathbb{M}_{n}\right) \\
d\left(\mathbb{M}_{n}, \mathbb{M}_{1}\right) & d\left(\mathbb{M}_{n}, \mathbb{M}_{2}\right) & \cdots & d\left(\mathbb{M}_{n}, \mathbb{M}_{n-1}\right) & 0
\end{array}\right]
$$


Step 2: The similarity $\operatorname{Sim}\left(\mathbb{M}_{i}, \mathbb{M}_{j}\right)$ between evidences is calculated as follows:

$$
\operatorname{Sim}\left(\mathbb{M}_{i}, \mathbb{M}_{j}\right)=1-d\left(\mathbb{M}_{i}, \mathbb{M}_{j}\right)
$$

And construct a similarity matrix $\mathcal{S}=\left(\operatorname{Sim}\left(\mathbb{M}_{i}, \mathbb{M}_{j}\right)\right)_{n \times n}$ as follows:

$$
\mathcal{S}=\left[\begin{array}{ccccc}
1 & \operatorname{Sim}\left(\mathbb{M}_{1}, \mathbb{M}_{2}\right) & \cdots & \operatorname{Sim}\left(\mathbb{M}_{1}, \mathbb{M}_{n-1}\right) & \operatorname{Sim}\left(\mathbb{M}_{1}, \mathbb{M}_{n}\right) \\
\operatorname{Sim}\left(\mathbb{M}_{2}, \mathbb{M}_{1}\right) & 1 & \cdots & \operatorname{Sim}\left(\mathbb{M}_{2}, \mathbb{M}_{n-1}\right) & \operatorname{Sim}\left(\mathbb{M}_{2}, \mathbb{M}_{n}\right) \\
\vdots & \vdots & \ddots & \vdots & \vdots \\
\operatorname{Sim}\left(\mathbb{M}_{n-1}, \mathbb{M}_{1}\right) & \operatorname{Sim}\left(\mathbb{M}_{n-1}, \mathbb{M}_{2}\right) & \cdots & 1 & \operatorname{Sim}\left(\mathbb{M}_{n-1}, \mathbb{M}_{n}\right) \\
\operatorname{Sim}\left(\mathbb{M}_{n}, \mathbb{M}_{1}\right) & \operatorname{Sim}\left(\mathbb{M}_{n}, \mathbb{M}_{2}\right) & \cdots & \operatorname{Sim}\left(\mathbb{M}_{n}, \mathbb{M}_{n-1}\right) & 1
\end{array}\right]
$$

Step 3: According to the similarity, the support was calculated as follows:

$$
\operatorname{Sup}\left(\mathbb{M}_{i}\right)=\sum_{j=1, i \neq 1}^{n} \operatorname{Sim}\left(\mathbb{M}_{i}, \mathbb{M}_{j}\right)=\left(\sum_{j=1}^{n} \operatorname{Sim}\left(\mathbb{M}_{i}, \mathbb{M}_{j}\right)\right)-1
$$

Step 4: The support Sup is normalized to calculate the weight:

$$
\operatorname{Crd}_{i}=\frac{\operatorname{Sup}\left(\mathbb{M}_{i}\right)}{\sum_{j=1}^{n} \operatorname{Sup}\left(\mathbb{M}_{j}\right)}
$$

Step 5: Combining weight and all CBBAs to calculate MACE. The calculation of MACE (A) is showed as follows:

$$
M A C E(A)=\sum_{i=1}^{n} \mathbb{M}_{i}(A) \cdot C r d_{i}
$$

The whole MACE can be obtained by analogy.

Step 6: Using GDRC let MACE fuses itself $n-1$ times.

To make the proposed method easier to understand, we give pseudo code here. The pseudocode of the proposed method algorithm is provided in Algorithm 1. 
Algorithm 1 Algorithm of the new multisource information fusion.

Input: The weighted CBBA, MACE;

The number of CBBAs before fusion, $n$;

Output: The final complex evidence combination result, $\mathbb{M}$;

1: for $i=1 ; i \leq n$ do

2: $\quad$ for $j=1 ; j \leq n$ do

3: $\quad$ Calculate the distance between different CBBAs pairs using Eq. (7-9);

4: $\quad$ end for

5: end for

6: As in Step 1, construct a $\mathcal{D}=\left(d\left(\mathbb{M}_{i}, \mathbb{M}_{j}\right)\right)_{n \times n}$ matrix and store the obtained $d\left(\mathbb{M}_{i}, \mathbb{M}_{j}\right)$ in it;

7: for $i=1 ; i \leq n$ do

8: $\quad$ for $j=1 ; j \leq n$ do

9: $\quad$ The similarity Sim between CBBAs and similarity matrix $\mathcal{S}$ are calculated by Eq. (10);

10: $\quad$ end for

11: end for

12: In addition, a matrix $\mathcal{S}=\left(\operatorname{Sim}\left(\mathbb{M}_{i}, \mathbb{M}_{j}\right)\right)_{n \times n}$ is constructed to store the similarity $\operatorname{Sim}\left(\mathbb{M}_{i}, \mathbb{M}_{j}\right)$ between pairs in Step 2;

13: for $i=1 ; i \leq n$ do

14: $\quad$ Supported sup is calculated according to Eq. (11);

15: end for

16: for $i=1 ; i \leq n$ do

17: The weight of each CBBAs is calculated according to Eq. (12); 


\section{8: end for}

19: for $i=1 ; i \leq n$ do

20: $\quad$ Calculate the MACE according to Eq. (13);

\section{1: end for}

22: Make $\mathbb{M}=\mathrm{MACE}$;

23: for $i=1 ; i \leq n-1$ do

24: $\quad$ Let $\mathbb{M}$ and MACE combine into a new CBBA using GDRC and assign it to $\mathbb{M}$;

25: end for

26: $\operatorname{return} \mathbb{M}$;

\section{A numerical example}

In this section, a numerical example is given to prove the feasibility of the proposed method. Several sets of CBBAs in Table 1 will be used for fusion.

Table 1: Several groups of CBBAs

\begin{tabular}{lllll}
\hline & \multicolumn{1}{c}{$\mathrm{A}$} & \multicolumn{1}{c}{$\mathrm{B}$} & \multicolumn{1}{c}{$\mathrm{C}$} & \multicolumn{1}{c}{$\mathrm{A}, \mathrm{C}$} \\
\hline $\mathbb{M}_{1}$ & $0.6041 e^{0.1161 i}$ & $0.1562 e^{-0.8761 i}$ & $0.0500 e^{-1.5708 i}$ & $0.3162 e^{0.3218 i}$ \\
$\mathbb{M}_{2}$ & $0.6041 e^{-0.3375 i}$ & $0.1414 e^{0.7854 i}$ & $0.0000 e^{0.0000 i}$ & $0.3448 e^{0.2942 i}$ \\
$\mathbb{M}_{3}$ & $0.0000 e^{0.0000 i}$ & $0.8062 e^{0.1244 i}$ & $0.2236 e^{-0.4636 i}$ & $0.0000 e^{0.0000 i}$ \\
$\mathbb{M}_{4}$ & $0.5689 e^{0.1767 i}$ & $0.1000 e^{0.0000 i}$ & $0.0000 e^{0.0000 i}$ & $0.3544 e^{-0.2861 i}$ \\
$\mathbb{M}_{5}$ & $0.6888 e^{0.4507 i}$ & $0.1000 e^{1.5708 i}$ & $0.1803 e^{-0.5880 i}$ & $0.2508 e^{-0.4101 i}$ \\
\hline
\end{tabular}

According to Eq. (5, 6), several groups of data are fused by GDRC. If two groups of CBBAs are fused, the two groups will be directly fused by GDRC. If there are $n$ groups of CBBAs, fusion $n-1$ times is needed. The first two sets of data are fused by GDRC. And then the fusion results were fused with 
the next group, same for the following groups. Eventually, the final results were obtained by fusing the fusion results to the last group.

The following will demonstrates the fusion method for $\mathbb{M}_{1} \oplus \mathbb{M}_{2} \oplus \mathbb{M}_{3} \oplus$ $\mathbb{M}_{4} \oplus \mathbb{M}_{5}$. At first, the distance can be obtained using the Eq. (7-9). Then, the distances between $\mathbb{M}_{1}$ and $\mathbb{M}_{2}$ shown follows:

$$
d\left(\mathbb{M}_{1}, \mathbb{M}_{2}\right)=\sqrt{\frac{\left\|\overrightarrow{\mathbb{M}}_{1}\right\|^{2}+\left\|\overrightarrow{\mathbb{M}}_{2}\right\|^{2}-2\left|\left\langle\overrightarrow{\mathbb{M}}_{1}, \overrightarrow{\mathbb{M}}_{2}\right\rangle\right|}{2}}=0.2142 .
$$

The value of each $\left(d\left(\mathbb{M}_{i}, \mathbb{M}_{j}\right)\right)$ is calculated from this. Then write it into the matrix $\mathcal{D}$, which showed as follows:

$$
\mathcal{D}=\left[\begin{array}{lllll}
0.0000 & 0.2142 & 0.6862 & 0.1187 & 0.2231 \\
0.2142 & 0.0000 & 0.6687 & 0.2290 & 0.3700 \\
0.6862 & 0.6687 & 0.0000 & 0.6730 & 0.7323 \\
0.1187 & 0.2290 & 0.6730 & 0.0000 & 0.2073 \\
0.2231 & 0.3700 & 0.7323 & 0.2073 & 0.0000
\end{array}\right] \text {. }
$$

According to Eq. (10), the similarity matrix $\mathcal{S}$ is obtained:

$$
\mathcal{S}=\left[\begin{array}{lllll}
1.0000 & 0.7858 & 0.3138 & 0.8813 & 0.7769 \\
0.7858 & 1.0000 & 0.3313 & 0.7710 & 0.6300 \\
0.3138 & 0.3313 & 1.0000 & 0.3270 & 0.2677 \\
0.8813 & 0.7710 & 0.3270 & 1.0000 & 0.7927 \\
0.7769 & 0.6300 & 0.2677 & 0.7927 & 1.0000
\end{array}\right]
$$

The support can be obtained by Eq. (11):

$$
\text { Sup }=[2.7578, \quad 2.5181, \quad 1.2398, \quad 2.7720, \quad 2.4673] \text {. }
$$

From Eq. (12) the weight factor is calculated according to the support degree:

$$
C r d=\left[\begin{array}{lllll}
0.2346, & 0.2142, & 0.1055, & 0.2358, & 0.2099
\end{array}\right] .
$$

The visualization of Crd is shown in Fig. 1. 


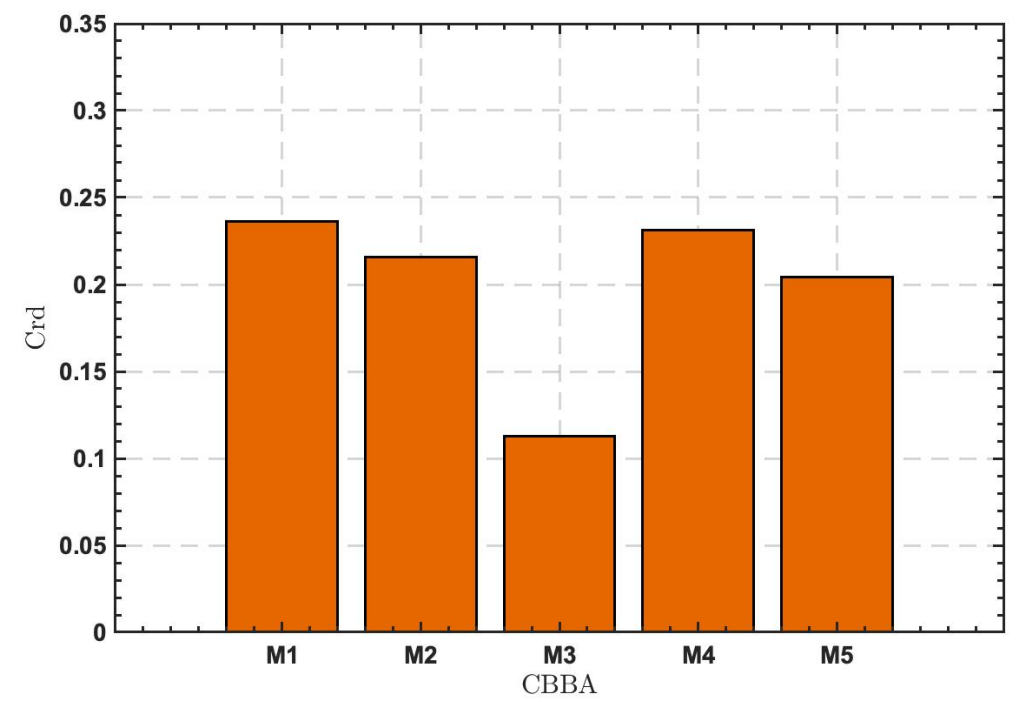

Figure 1: Crd visualization based on fusion of five CBBAs

MACE is calculated by Eq. (13):

$$
M A C E=\left[\begin{array}{llll}
0.5285 e^{0.1140 i}, & 0.1538 e^{-0.1119 i}, & 0.0681 e^{-0.6886 i}, & 0.2695 e^{0.0012 i}
\end{array}\right] .
$$

Finally, according to the algorithm in Section 3.3, let MACE perform $5-1=4$ times of GDRC. Let $\mathbb{M}$ be the final fusion result of these five CBBAs groups:

$$
\mathbb{M}=\left[\begin{array}{llll}
0.9118 e^{-0.0556 i}, & 0.0917 e^{0.4427 i}, & 0.01103 e^{1.2174 i}, & 0.0031 e^{0.2915 i}
\end{array}\right]
$$

If we use $\mathbb{R}_{1}$ stands for $\mathbb{M}_{1} \oplus \mathbb{M}_{2}, \mathbb{R}_{2}$ stands for $\mathbb{M}_{1} \oplus \mathbb{M}_{2} \oplus \mathbb{M}_{3}$, $\mathbb{R}_{3}$ stands for $\mathbb{M}_{1} \oplus \mathbb{M}_{2} \oplus \mathbb{M}_{3} \oplus \mathbb{M}_{4}$ and $\mathbb{R}_{4}$ stands for $\mathbb{M}_{1} \oplus \mathbb{M}_{2} \oplus \mathbb{M}_{3} \oplus \mathbb{M}_{4} \oplus$ $\mathbb{M}_{5}$. The others fusion results of GDRC and proposed method are shown in Table 2 . 
Table 2: Two fusion method results

\begin{tabular}{cccccc}
\hline & Evidence & $\mathbb{R}_{1}$ & $\mathbb{R}_{2}$ & $\mathbb{R}_{3}$ & $\mathbb{R}_{4}$ \\
\hline \multirow{4}{*}{ GDRC } & $\mathbb{M}(A)$ & $0.8016 e^{0.0597 i}$ & $0.0000 e^{0.0000 i}$ & $0.0000 e^{0.0000 i}$ & $0.0000 e^{0.0000 i}$ \\
& $\mathbb{M}(B)$ & $0.0950 e^{-0.0369 i}$ & $0.8804 e^{-0.0664 i}$ & $0.9068 e^{-0.0595 i}$ & $0.8615 e^{-0.0495 i}$ \\
& $\mathbb{M}(C)$ & $0.0187 e^{1.3336 i}$ & $0.1348 e^{0.4481 i}$ & $0.1091 e^{0.5170 i}$ & $0.1460 e^{0.2962 i}$ \\
& $\mathbb{M}(A, C)$ & $0.1184 e^{-0.5559 i}$ & $0.0000 e^{0.0000 i}$ & $0.0000 e^{0.0000 i}$ & $0.0000 e^{0.0000 i}$ \\
\hline \multirow{2}{*}{ Proposed } & $\mathbb{M}(A)$ & $0.8085 e^{0.0663 i}$ & $0.7097 e^{0.1196 i}$ & $0.8654 e^{0.0168 i}$ & $0.9640 e^{-0.0302 i}$ \\
method & $\mathbb{M}(B)$ & $0.0851 e^{-0.0882 i}$ & $0.2542 e^{-0.3016 i}$ & $0.1146 e^{-0.1532 i}$ & $0.0364 e^{0.5743 i}$ \\
& $\mathbb{M}(C)$ & $0.0184 e^{1.3681 i}$ & $0.0295 e^{0.5288 i}$ & $0.0010 e^{0.7783 i}$ & $0.0080 e^{1.3562 i}$ \\
& $\mathbb{M}(A, C)$ & $0.1227 e^{-0.5487 i}$ & $0.0363 e^{-0.7250 i}$ & $0.0148 e^{-0.2727 i}$ & $0.0043 e^{0.3588 i}$ \\
\hline
\end{tabular}

Because the complex numbers cannot compared by size, so the computer cannot judge the fusion result directly. We can make the final judgment by calculating the complex module lengths. The result of the module lengths are shown in Table 3.

Table 3: Module length of numerical example results

\begin{tabular}{cccccc}
\hline & Evidence & $\mathbb{R}_{1}$ & $\mathbb{R}_{2}$ & $\mathbb{R}_{3}$ & $\mathbb{R}_{4}$ \\
\hline \multirow{4}{*}{ GDRC } & $\mathbb{M}(A)$ & 0.8016 & 0.0000 & 0.0000 & 0.0000 \\
& $\mathbb{M}(B)$ & 0.0950 & 0.8804 & 0.9068 & 0.8615 \\
& $\mathbb{M}(C)$ & 0.0187 & 0.1348 & 0.1091 & 0.1460 \\
Proposed & $\mathbb{M}(A, C)$ & 0.1184 & 0.0000 & 0.0000 & 0.0000 \\
\hline \multirow{3}{*}{ method } & $\mathbb{M}(A)$ & 0.8085 & 0.7097 & 0.8654 & 0.9640 \\
& $\mathbb{M}(C)$ & 0.0851 & 0.2542 & 0.1146 & 0.0364 \\
& $\mathbb{M}(A, C)$ & 0.0184 & 0.0295 & 0.0100 & 0.0080 \\
\hline
\end{tabular}

The module lengths in Table 3 are visualized in Fig. 2.

As shown in Table 4, among the five CBBAs, the frame of $\mathbb{M}_{3}$ is deficiency, 

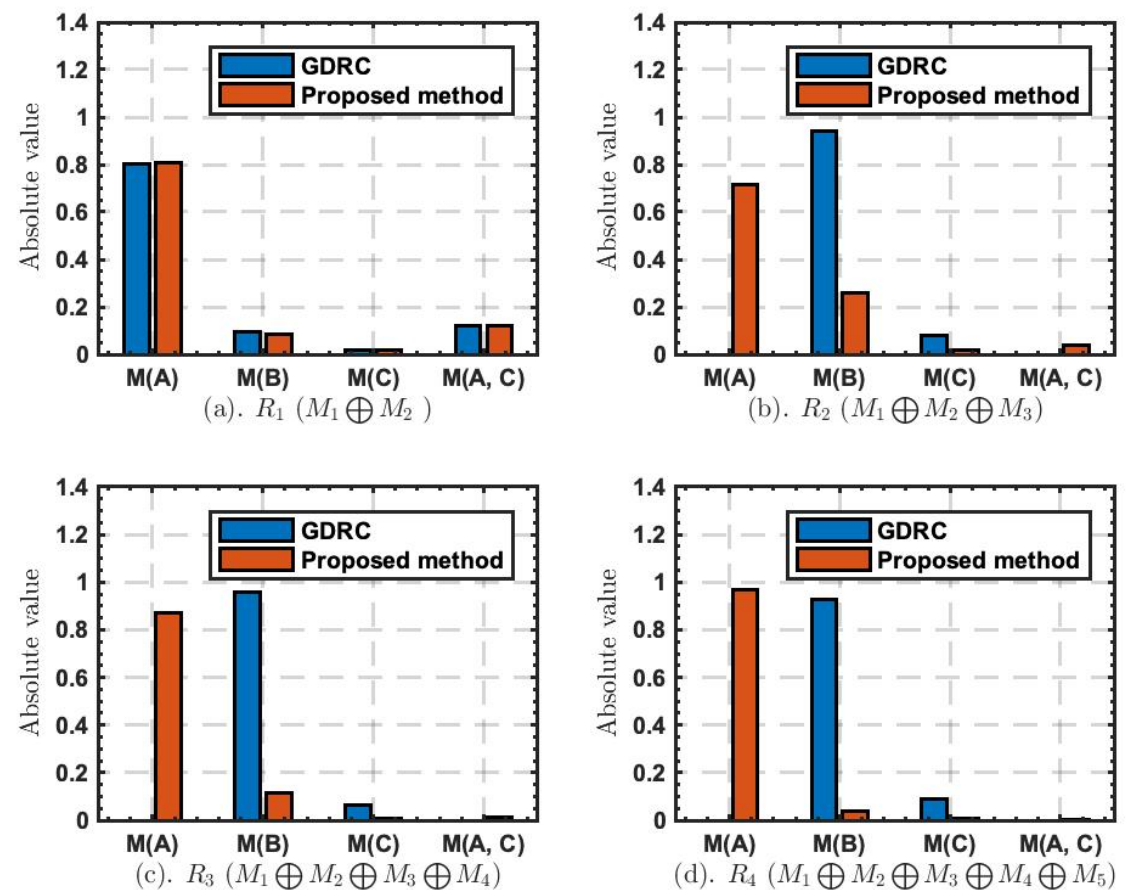

Figure 2: The flow chart of PICCA-based gesture recognition

represented by $\mathbb{M}_{3}$ on the diagram with 0 supports for $\{A\}$ and $\{A, C\}$. However, $\mathbb{M}_{1}, \mathbb{M}_{2}, \mathbb{M}_{4}$ and $\mathbb{M}_{5}$ have higher value which is 0.6041, 0.6041, $0.5689,0.6888$ to support event $\{A\}$ respectively. The first fusion result as shown in Fig. 2(a), from then we can illustrate that the results from GDRC and the proposed method are the same because of the less difference between $\mathbb{M}_{1}$ and $\mathbb{M}_{2}$, which can clarify the deficiency of the proposed method. When adding completely conflict fusion result even the frame of $\mathbb{M}_{3}$ is lost, the proposed method still obtains a higher support 0.7079 for event $\{A\}$ showed in Fig. 2(b), which means the fusion results are less affected by $\mathbb{M}_{3}$. However, GDRC is more supportive towards event $\{B\}$, which obeys a support up to 
0.8804. It is obvious that GDRC's result goes against the facts. The reason why the proposed method occupies higher reliability is that the majority of CBBAs support event $\{A\}$, however, the GDRC attains 0 for event $\{A\}$. In Fig. 2(c) and Fig. 2(d), we garner two other sets of CBBAs that are similar to $\mathbb{M}_{1}, \mathbb{M}_{2}, \mathbb{M}_{4}$ and $\mathbb{M}_{5}$ additionally. In spite of the addition of $\mathbb{M}_{3}$, the fusion results indicate that as the support value towards event $\{A\}$ of $\mathbb{M}_{4}$ and $\mathbb{M}_{5}$ changed, the support of event $\{A\}$ from GDRC remains 0, it against the fact clearly. However, the proposed method calculates the distance between difference evidence and allocates different weights according to the distance. It can be inferred from the first group and fifth group in Fig. 1 that during the fusion of CBBAs the proposed method assigns weight, called $C r d$, to $\mathbb{M}_{3}$ is 0.1055 which is far less than other CBBAs owing to the wide difference from other CBBAs. In this way, the influence from $\mathbb{M}_{3}$ to fusion results has been decreased, which makes the results more dependable.

\section{Application}

In this section, we review a medical diagnosis problem to verify the feasibility of the proposed method proposed in this paper.

\subsection{Problem statement}

On the basis of literature [61, 76], we use the data in Table 4. Consider a medical diagnosis decision problem with three diseases, $E=\left\{\left\{E_{1}\right.\right.$ : Viralfever $\},\left\{E_{2}:\right.$ Malaria $\},\left\{E_{3}:\right.$ Typhoid $\left.\}\right\}$. One patient had a disease in $E_{1}, E_{2}$ and $E_{3}$. We have 5 experts individually assessing the patient's condition and thus determine which condition the patient is most likely to have. The expert's evaluation value is modeled in Table 4 as a complex number 
of basic belief assignments. Among them, the threshold value $\theta$ is set as 0.5 for the decisionmaking here. The medical diagnosis decision is based on the evaluation of five experts to diagnose the disease of the patient in $E_{1}, E_{2}$ and $E_{3}$.

Table 4: medical diagnosis

\begin{tabular}{ccccc}
\hline & \multicolumn{4}{c}{ Diseases } \\
\cline { 2 - 5 } CBBAs & Viral fever & Malaria & Typhoid & Uncertain situation \\
\cline { 2 - 5 } & $\left\{E_{1}\right\}$ & $\left\{E_{2}\right\}$ & $\left\{E_{3}\right\}$ & $\{\theta\}$ \\
\hline $\mathbb{M}_{1}$ & $0.5721 e^{0.1212 i}$ & $0.1095 e^{1.3008 i}$ & $0.1001 e^{0.3034 i}$ & $0.2642 e^{-0.5072 i}$ \\
$\mathbb{M}_{2}$ & $0.0740 e^{1.2191 i}$ & $0.1607 e^{1.3880 i}$ & $0.6961 e^{0.0425 i}$ & $0.1763 e^{-0.8151 i}$ \\
$\mathbb{M}_{3}$ & $0.5321 e^{0.0188 i}$ & $0.1000 e^{1.2702 i}$ & $0.1500 e^{0.8737 i}$ & $0.3164 e^{-0.5106}$ \\
$\mathbb{M}_{4}$ & $0.5703 e^{0.0338 i}$ & $0.1005 e^{1.2708 i}$ & $0.0744 e^{0.7293 i}$ & $0.2954 e^{-0.3403 i}$ \\
$\mathbb{M}_{5}$ & $0.4816 e^{0.0817 i}$ & $0.1068 e^{1.3863 i}$ & $0.0606 e^{0.3468 i}$ & $0.3668 e^{-0.2185 i}$ \\
\hline
\end{tabular}

\subsection{Fusion results}

It can be seen that in Table 4, there is a big gap between the measurement results of $\mathbb{M}_{4}$ and other data, if it is impossible to judge whether the $\mathbb{M}_{4}$ sensor is abnormal, which is likely to have an impact on the results. We use $\mathbb{R}_{1}$ stands for $\mathbb{M}_{1} \oplus \mathbb{M}_{2}, \mathbb{R}_{2}$ stands for $\mathbb{M}_{1} \bigoplus \mathbb{M}_{2} \bigoplus \mathbb{M}_{3}, \mathbb{R}_{3}$ stands for $\mathbb{M}_{1} \oplus \mathbb{M}_{2} \oplus \mathbb{M}_{3} \oplus \mathbb{M}_{4}$ and $\mathbb{R}_{4}$ stands for $\mathbb{M}_{1} \oplus \mathbb{M}_{2} \oplus \mathbb{M}_{3} \oplus \mathbb{M}_{4} \oplus \mathbb{M}_{5}$. The results of the proposed method and GDRC are calculated in Table 5.

Similarly, the module lengths of each CBBAs is calculated in Table 6 which are convenient for later decision.

The results in Table 6 are visualized and the bar chart is drawn, as shown in Fig. 3. 
Table 5: Fusion results of two methods

\begin{tabular}{cccccc}
\hline \multirow{2}{*}{$\begin{array}{c}\text { Evidence } \\
\text { combination }\end{array}$} & \multicolumn{5}{c}{ Diseases } \\
\cline { 3 - 6 } & \cline { 2 - 5 } & Viral fever & Malaria & Typhoid & Uncertain situation \\
\cline { 2 - 6 }$\left\{E_{1}\right\}$ & $\mathbb{R}_{1}$ & $0.7559 e^{0.0224 i}$ & $0.0864 e^{-0.1021 i}$ & $0.0970 e^{-0.4954 i}$ & $0.1025 e^{0.7764 i}$ \\
GDRC & $\mathbb{R}_{2}$ & $0.4184 e^{0.0069 i}$ & $0.1389 e^{0.0684 i}$ & $0.4315 e^{-0.1641 i}$ & $0.0606 e^{1.2814 i}$ \\
& $\mathbb{R}_{3}$ & $0.6433 e^{0.0039 i}$ & $0.0996 e^{0.1401 i}$ & $0.2584 e^{-0.1833 i}$ & $0.0310 e^{1.4412 i}$ \\
& $\mathbb{R}_{4}$ & $0.7777 e^{-0.0167 i}$ & $0.0691 e^{0.2306 i}$ & $0.1561 e^{-0.1221 i}$ & $0.0162 e^{1.5646 i}$ \\
\hline \multirow{2}{*}{ Proposed } & $\mathbb{R}_{2}$ & $0.6677 e^{-0.0321 i}$ & $0.0826 e^{0.1287 i}$ & $0.2395 e^{-0.1180 i}$ & $0.0421 e^{1.2017 i}$ \\
method & $\mathbb{R}_{3}$ & $0.8559 e^{-0.0240 i}$ & $0.0445 e^{0.2521 i}$ & $0.0983 e^{-0.0713 i}$ & $0.0168 e^{1.3792 i}$ \\
& $\mathbb{R}_{4}$ & $0.9236 e^{-0.0217 i}$ & $0.0281 e^{0.3633 i}$ & $0.4935 e^{0.0426 i}$ & $0.0080 e^{1.4574 i}$ \\
\hline
\end{tabular}

Table 6: Module lengths of combined results

\begin{tabular}{cccccc}
\hline \multirow{2}{*}{$\begin{array}{c}\text { Evidence } \\
\text { combination }\end{array}$} & \multicolumn{4}{c}{ Diseases } \\
\cline { 2 - 6 } & Viral fever & Malaria & Typhoid & Uncertain situation \\
\cline { 2 - 6 } & $\mathbb{R}_{1}$ & 0.7559 & 0.0864 & 0.0970 & 0.1025 \\
\hline \multirow{2}{*}{ GDRC } & $\mathbb{R}_{2}$ & 0.4184 & 0.1389 & 0.4315 & 0.0606 \\
& $\mathbb{R}_{3}$ & 0.6433 & 0.0996 & 0.2584 & 0.0310 \\
& $\mathbb{R}_{4}$ & 0.7777 & 0.0691 & 0.1561 & 0.0162 \\
\hline \multirow{2}{*}{ Proposed } & $\mathbb{R}_{2}$ & 0.7567 & 0.0864 & 0.0965 & 0.1035 \\
method & $\mathbb{R}_{3}$ & 0.8559 & 0.0826 & 0.2395 & 0.0421 \\
& $\mathbb{R}_{5}$ & 0.9236 & 0.0281 & 0.4935 & 0.0168 \\
\hline
\end{tabular}




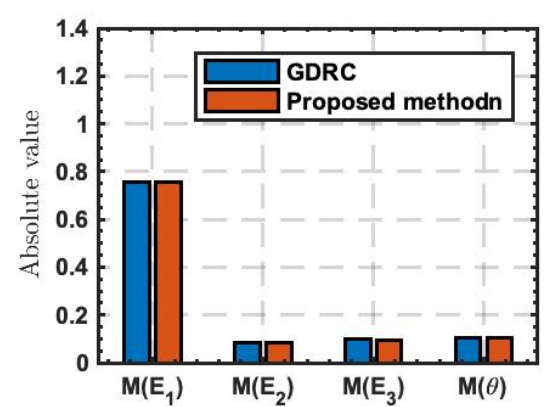

(a). $R_{1}\left(M_{1} \oplus M_{2}\right)$

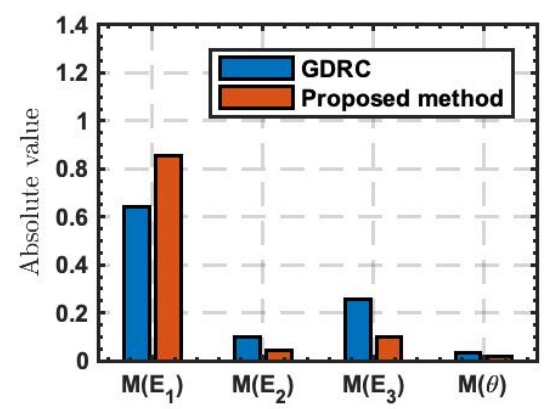

(c). $R_{3}\left(M_{1} \oplus M_{2} \oplus M_{3} \oplus M_{4}\right)$

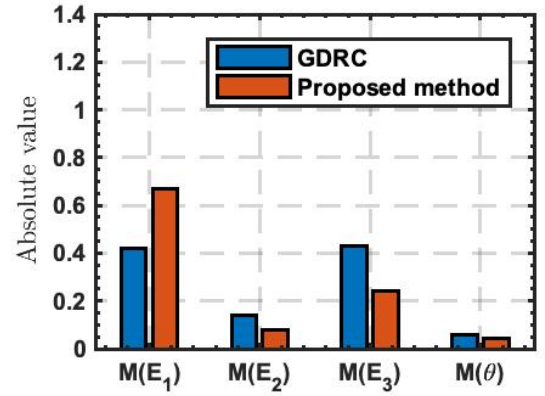

(b). $R_{2}\left(M_{1} \oplus M_{2} \oplus M_{3}\right)$

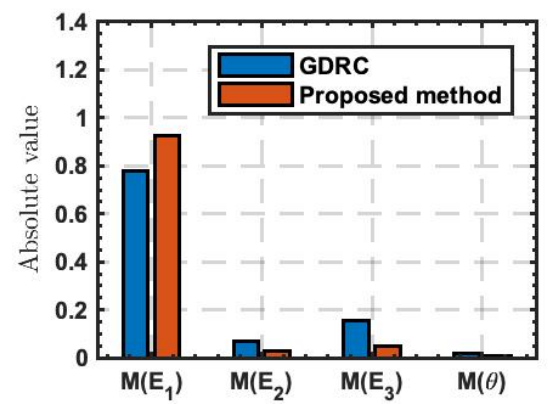

(d). $R_{4}\left(M_{1} \oplus M_{2} \oplus M_{3} \oplus M_{4} \oplus M_{5}\right)$

Figure 3: Visualization of the results of the medical diagnosis problem

\subsection{Discussion}

As shown in Table 4 , it is obvious that $\mathbb{M}_{3}$ conflicts with other evidence $\mathbb{M}_{1}, \mathbb{M}_{2}, \mathbb{M}_{4}$ and $\mathbb{M}_{5}$. For disease $E_{1}$ : Viral fever. $\mathbb{M}_{1}, \mathbb{M}_{2}, \mathbb{M}_{4}$ and $\mathbb{M}_{5}$ have higher absolute values $0.5721,0.5321,0.5703$ and 0.4816 to support it. But $\mathbb{M}_{3}$ only have absolute value 0.0740 to support $E_{1}$.

From the fusion results in Table 5 and Fig. 3. It is obvious that in Fig. 3(a) when the first two CBBAs $\mathbb{M}_{1}$ and $\mathbb{M}_{2}$ are fused, the result of GDRC and proposed method is similar. This is because $\mathbb{M}_{1}$ and $\mathbb{M}_{2}$ are not conflicting. Hence, when there is no conflict evidence fusion, the proposed method and GDRC fusion result is same, which can reflect the feasibility of 
the proposed method. When $\mathbb{M}_{1}, \mathbb{M}_{2}$ and $\mathbb{M}_{3}$ fusion (Fig. 3(b)), the results of GDRC and proposed method are very different due to the participance of $\mathbb{M}_{3}$, where GDRC has the highest absolute values 0.4315 to support $E_{3}$ : Typhoid, the proposed method has higher absolute values 0.6677 to support $E_{1}$ : Viral faver. The consequence of above fusion results are clairfied. There are two of the three CBBAs support $E_{1}$, however, GDRC supports $E_{3}$. The proposed method is different from the diseases supported by GDRC because the proposed method calculates the distance between each CBBA, of which $\mathbb{M}_{3}$ is in great conflict with other CBBAs. $\mathbb{M}_{3}$ has a huge gap distance from other CBBAs. According to the distance, the allocation of weight in $\mathbb{M}_{3}$ is decreased sharply, which causes a little impact on the results. In this way, the proposed method still supports $E_{1}$. In Fig. 3(c)and Fig. 3(d), although both GDRC and proposed method support $E_{1}$, obviously when the first four

groups fused with all CBBAs, the support of $E_{1}$, which GDRC is less than the proposed method. This is because the proposed method assigns weight to all CBBAs according to CED, which measures the difference between $\mathbb{M}_{3}$ and other CBBAs, results $\mathbb{M}_{3}$ had little effect corespondingly. Proposed method for $E_{1}$ is more convincing.

\section{Conclusions}

The classical D-S theory is often used in such uncertain problems in the range of real numbers situation. Most probability in life is expressed in real number, but some problems in life, such as medical sensors, mechanical detection and other signals are often not real. Complex evidence theory has significally beneficial expression to uncertainty and imprecision. But 
the complex evidence theory will inevitably encounter the problem of evidence conflict leading to the wrong fusion results. In this paper, a new multisource information fusion for CBBAs is proposed to make the decision more accurate. This fusion can effectively solve the problem of incomplete identification framework and it also can reduce the influence of sensor error effectively. Then, the effectiveness and feasibility of this proposed method are verified by the numerical example and the application.

In the future research, we will further study the process of weight allocation. In addition, we hope to expand the research methods to get more applications.

\section{Compliance with Ethical Standards}

Funding: This study is partially supported by the National Natural Science Foundation of China (No. 62003280).

Conflict of Interest: Author Huaping He declares that he has no confict of interest. Author Liting He declares that she has no conflict of interest. Author Fuyuan Xiao declares that she has no confict of interest.

Ethical approval: This article does not contain any studies with human participants or animals performed by any of the authors.

\section{References}

[1] D. Meng, T. Xie, P. Wu, S.-P. Zhu, Z. Hu, Y. Li, Uncertainty-based design and optimization using first order saddle point approximation method for multidisciplinary engineering systems, ASCE-ASME Jour- 
nal of Risk and Uncertainty in Engineering Systems, Part A: Civil Engineering 6 (3) (2020) 04020028.

[2] Y. Deng, Uncertainty measure in evidence theory, SCIENCE CHINA Information Sciences 63 (11) (2020) 210201.

[3] F. Cuzzolin, The Geometry of Uncertainty - The Geometry of Imprecise Probabilities, Artificial Intelligence: Foundations, Theory, and Algorithms, Springer, 2021.

[4] X. Wang, Y. Song, Uncertainty measure in evidence theory with its applications, Applied Intelligence 48 (7) (2018) 1672-1688.

[5] T. Wen, K. H. Cheong, The fractal dimension of complex networks: A review, Information Fusion 73 (2021) 87-102.

[6] Y. Deng, Information volume of mass function, International Journal of Computers Communications \& Control 15 (6) (2020) 3983.

[7] J. Zhou, X. Su, H. Qian, Risk assessment on offshore photovoltaic power generation projects in china using d numbers and anp, IEEE Access 8 (2020) 144704-144717.

[8] H. Mo, Y. Deng, A new aggregating operator for linguistic information based on d numbers, International Journal of Uncertainty, Fuzziness and Knowledge-Based Systems 24 (6) (2016) 831-846.

[9] Q. Liu, Y. Tian, B. Kang, Derive knowledge of Z-number from the perspective of Dempster-Shafer evidence theory, Engineering Applications of Artificial Intelligence 85 (2019) 754-764. 
[10] Y. Tian, L. Liu, X. Mi, B. Kang, ZSLF: A new soft likelihood function based on Z-numbers and its application in expert decision system, IEEE Transactions on Fuzzy Systems (2020) DOI: 10.1109/TFUZZ.2020.2997328.

[11] L. Pan, Y. Deng, Probability transform based on the ordered weighted averaging and entropy difference, International Journal of Computers Communications \& Control 15 (4) (2020) 3743.

[12] F. Xiao, EFMCDM: Evidential fuzzy multicriteria decision making based on belief entropy, IEEE Transactions on Fuzzy Systems 28 (7) (2020) 1477-1491.

[13] S. Babajanyan, A. Allahverdyan, K. H. Cheong, Energy and entropy: Path from game theory to statistical mechanics, Physical Review Research 2 (4) (2020) 043055.

[14] F. Xiao, On the maximum entropy negation of a complex-valued distribution, IEEE Transactions on Fuzzy Systems (2020) DOI: 10.1109/TFUZZ.2020.3016723.

[15] H. Liao, Z. Ren, R. Fang, A Deng-entropy-based evidential reasoning approach for multi-expert multi-criterion decision-making with uncertainty, International Journal of Computational Intelligence Systems 13 (1) (2020) 1281-1294.

[16] A. Dempster, Upper and lower probabilities induced by a multivalued mapping, The Annals of Mathematical Statistics 38 (2) (1967) 325-339. 
[17] G. Shafer, A mathematical theory of evidence, Vol. 42, Princeton university press, 1976.

[18] R. R. Yager, Generalized Dempster-Shafer structures, IEEE Transactions on Fuzzy Systems 27 (3) (2019) 428-435.

[19] X. Feng, Z. Xiao, X. Wang, B. Zhong, Peer-to-peer lending platform selection using intuitionistic fuzzy soft set and d-s theory of evidence, International Journal of Uncertainty, Fuzziness and Knowledge-Based Systems 27 (1) (2019) 1-17.

[20] X. Deng, W. Jiang, On the negation of a Dempster-Shafer belief structure based on maximum uncertainty allocation, Information Sciences 516 (2020) 346-352.

[21] J. Ye, J. Zhan, W. Ding, H. Fujita, A novel fuzzy rough set model with fuzzy neighborhood operators, Information Sciences 544 (2021) 266-297.

[22] H. Garg, D. Rani, Some results on information measures for complex intuitionistic fuzzy sets, International Journal of Intelligent Systems 34 (10) (2019) 2319-2363.

[23] K. Zhang, J. Zhan, W. Z. Wu, On multi-criteria decision-making method based on a fuzzy rough set model with fuzzy -neighborhoods, IEEE Transactions on Fuzzy Systems (2020) 10.1109/TFUZZ.2020.3001670.

[24] M. G. Barbera-Marine, Y. Laumann, L. Fabregat-Aibar, Analysis of fuzzy beta coefficients. evidence from the mexican stock market, International Journal of Uncertainty Fuzziness and Knowledge-Based Systems 26 (DEC.SUPPL.1) (2018) 59-69. 
[25] J. C. Alcantud, F. Feng, R. Yager, An N-soft set approach to rough sets, IEEE Transactions on Fuzzy Systems (2019) DOI: 10.1109/TFUZZ.2019.2946526.

[26] F. Feng, J. Cho, W. Pedrycz, H. Fujita, T. Herawan, Soft set based association rule mining, Knowledge-Based Systems 111 (2016) 268-282.

[27] L. Fei, Y. Feng, L. Liu, Evidence combination using OWA-based soft likelihood functions, International Journal of Intelligent Systems 34 (9) (2019) 2269-2290.

[28] F. Xiao, Z. Cao, A. Jolfaei, A novel conflict measurement in decision making and its application in fault diagnosis, IEEE Transactions on Fuzzy Systems (2020) DOI: 10.1109/TFUZZ.2020.3002431.

[29] J. Mi, Y.-F. Li, W. Peng, H.-Z. Huang, Reliability analysis of complex multi-state system with common cause failure based on evidential networks, Reliability Engineering \& System Safety 174 (2018) 71-81.

[30] X. Deng, Y. Yang, J. Yang, A novel discrete evidence fusion approach by considering the consistency of belief structures, Engineering Applications of Artificial Intelligence 96 (2020) 103994.

[31] Z. Liu, Q. Pan, J. Dezert, J.-W. Han, Y. He, Classifier fusion with contextual reliability evaluation, IEEE Transactions on Cybernetics 48 (5) (2018) 1605-1618.

[32] X. Xu, J. Zheng, J.-b. Yang, D.-l. Xu, Y.-w. Chen, Data classification using evidence reasoning rule, Knowledge-Based Systems 116 (2017) 144151. 
[33] Z.-G. Liu, L.-Q. Huang, K. Zhou, T. Denoeux, Combination of transferable classification with multisource domain adaptation based on evidential reasoning, IEEE Transactions on Neural Networks and Learning Systems (2020) DOI: 10.1109/TNNLS.2020.2995862.

[34] P. Liu, M. Shen, F. Teng, B. Zhu, L. Rong, Y. Geng, Double hierarchy hesitant fuzzy linguistic entropy-based TODIM approach using evidential theory, Information Sciences 547 (2021) 223-243.

[35] X. Xu, H. Xu, C. Wen, J. Li, P. Hou, J. Zhang, A belief rule-based evidence updating method for industrial alarm system design, Control Engineering Practice 81 (2018) 73-84.

[36] Z. Cao, C.-H. Chuang, J.-K. King, C.-T. Lin, Multi-channel EEG recordings during a sustained-attention driving task, Scientific Data 6 (2019) DOI: $10.1038 / \mathrm{s} 41597-019-0027-4$.

[37] J. Ma, Y. Ma, C. Li, Infrared and visible image fusion methods and applications: a survey, Inf. Fusion 45 (2019) 153-178.

[38] H. Fujita, Y.-C. Ko, A heuristic representation learning based on evidential memberships: Case study of UCI-SPECTF, International Journal of Approximate Reasoning 120 (2020).

[39] K. H. Cheong, J. M. Koh, M. C. Jones, Paradoxical survival: Examining the parrondo effect across biology, BioEssays 41 (6) (2019) 1900027.

[40] L. Fan, Y. Deng, Determine the number of unknown targets in Open World based on Elbow method, IEEE Transactions on Fuzzy Systems 29 (5) (2021) 986-995. 
[41] Y. Pan, L. Zhang, Z. Li, L. Ding, Improved fuzzy bayesian networkbased risk analysis with interval-valued fuzzy sets and D-S evidence theory, IEEE Transactions on Fuzzy Systems (2019) DOI: 10.1109/TFUZZ.2019.2929024.

[42] C. Fu, W. Chang, S. Yang, Multiple criteria group decision making based on group satisfaction, Information Sciences 518 (2020) 309-329.

[43] J. Zhan, H. Jiang, Y. Yao, Three-way multi-attribute decision-making based on outranking relations, IEEE Transactions on Fuzzy Systems (2020) 10.1109/TFUZZ.2020.3007423.

[44] W. Wang, J. Zhan, C. Zhang, Three-way decisions based multi-attribute decision making with probabilistic dominance relations, Information Sciences 559 (3) (2021) 10.1016/j.ins.2021.01.028.

[45] C. Fu, B. Hou, W. Chang, N. Feng, S. Yang, Comparison of evidential reasoning algorithm with linear combination in decision making, International Journal of Fuzzy Systems 22 (2) (2020) 686-711.

[46] H. Wang, Y.-P. Fang, E. Zio, Risk assessment of an electrical power system considering the influence of traffic congestion on a hypothetical scenario of electrified transportation system in new york state, IEEE Transactions on Intelligent Transportation Systems 22 (1) (2021) 142155.

[47] J. Yang, S. Li, Z. Xu, H. Liu, W. Yao, An understandable way to extend the ordinary linear order on real numbers to a linear order on 
interval numbers, IEEE Transactions on Fuzzy Systems (2020) DOI: 10.1109/TFUZZ.2020.3006557.

[48] Z. Liu, X. Zhang, J. Niu, J. Dezert, Combination of classifiers with different frames of discernment based on belief functions, IEEE Transactions on Fuzzy Systems (2020) DOI: 10.1109/TFUZZ.2020.2985332.

[49] L. Fei, Y. Feng, L. Liu, On Pythagorean fuzzy decision making using soft likelihood functions, International Journal of Intelligent Systems 34 (12) (2019) 3317-3335.

[50] W. Jiang, K. Huang, J. Geng, X. Deng, Multi-scale metric learning for few-shot learning, IEEE Transactions on Circuits and Systems for Video Technology (2020) DOI: 10.1109/TCSVT.2020.2995754.

[51] H. Zhang, Y. Deng, Entropy Measure for Orderable Sets, Information Sciences 561 (2021) 141-151.

[52] J. Zhao, Y. Deng, Complex network modeling of evidence theory, IEEE Transactions on Fuzzy Systems (2020) 10.1109/TFUZZ.2020.3023760.

[53] M. Tang, H. Liao, E. Herrera-Viedma, C. P. Chen, W. Pedrycz, A dynamic adaptive subgroup-to-subgroup compatibility-based conflict detection and resolution model for multicriteria large-scale group decision making, IEEE Transactions on Cybernetics (2020) DOI: 10.1109/TCYB.2020.2974924.

[54] Y. Song, J. Zhu, L. Lei, X. Wang, A self-adaptive combination method for temporal evidence based on negotiation strategy, SCIENCE CHINA Information Sciences (2020) 10.1007/s11432-020-3045-5. 
[55] M. Zhou, X.-B. Liu, Y.-W. Chen, J.-B. Yang, Evidential reasoning rule for MADM with both weights and reliabilities in group decision making, Knowledge-Based Systems 143 (2018) 142-161.

[56] X. Gao, L. Pan, Y. Deng, Quantum Pythagorean Fuzzy Evidence Theory (QPFET): A Negation of Quantum Mass Function View, IEEE Transactions on Fuzzy Systems (2021) 10.1109/TFUZZ.2021.3057993.

[57] X. Su, L. Li, H. Qian, S. Mahadevan, Y. Deng, A new rule to combine dependent bodies of evidence, Soft Computing 23 (20) (2019) 9793-9799.

[58] H. Wang, X. Deng, W. Jiang, J. Geng, A new belief divergence measure for dempster-shafer theory based on belief and plausibility function and its application in multi-source data fusion, Engineering Applications of Artificial Intelligence 97 (2021) 104030.

[59] Z. Liu, Y. Liu, J. Dezert, F. Cuzzolin, Evidence combination based on credal belief redistribution for pattern classification, IEEE Transactions on Fuzzy Systems 28 (4) (2020) 618-631.

[60] X. Gao, Y. Deng, Quantum model of mass function, International Journal of Intelligent Systems 35 (2) (2020) 267-282.

[61] F. Xiao, Generalization of Dempster-Shafer theory: A complex mass function, Applied Intelligence 50 (10) (2019) 3266-3275.

[62] Y. Deng, Information volume of mass function, International Journal of Computers Communications \& Control 15 (6) (2020) 3983. 
[63] F. Xiao, CEQD: A complex mass function to predict interference effects, IEEE Transactions on Cybernetics (2020) DOI: 10.1109/TCYB.2020.3040770.

[64] H. L. Huang, Y. Guo, An improved correlation coefficient of intuitionistic fuzzy sets, Journal of Intelligent Systems (2017).

[65] F. Xiao, GIQ: A generalized intelligent quality-based approach for fusing multi-source information, IEEE Transactions on Fuzzy Systems (2020) DOI: 10.1109/TFUZZ.2020.2991296.

[66] P. Liu, X. Zhang, W. Pedrycz, A consensus model for hesitant fuzzy linguistic group decision-making in the framework of Dempster-Shafer evidence theory, Knowledge-Based Systems 212 (2020) 106559.

[67] F. Xiao, A distance measure for intuitionistic fuzzy sets and its application to pattern classification problems, IEEE Transactions on Systems, Man, and Cybernetics: Systems (2019) DOI: 10.1109/TSMC.2019.2958635.

[68] J. Deng, Y. Deng, Information volume of fuzzy membership function, International Journal of Computers Communications \& Control 16 (1) (2021) 4106.

[69] M. Zhou, X.-B. Liu, Y.-W. Chen, X.-F. Qian, J.-B. Yang, J. Wu, Assignment of attribute weights with belief distributions for MADM under uncertainties, Knowledge-Based Systems 189 (2020) 105110.

[70] F. Xiao, Generalized belief function in complex evidence theory, Journal of Intelligent \& Fuzzy Systems 38 (4) (2020) 3665-3673. 
[71] F. Liu, X. Gao, J. Zhao, Y. Deng, Generalized belief entropy and its application in identifying conflict evidence, IEEE Access 7 (1) (2019) 126625-126633.

[72] A. L. Jousselme, D. Grenier, éloi Bossé, A new distance between two bodies of evidence, Information Fusion 2 (2) (2001) 91-101.

[73] Y. Yang, D. Han, A new distance-based total uncertainty measure in the theory of belief functions, Knowledge-Based Systems 94 (2016) 114-123.

[74] D. Han, J. Dezert, Y. Yang, Belief interval-based distance measures in the theory of belief functions, IEEE Transactions on Systems, Man, and Cybernetics: Systems 48 (6) (2016) 833-850.

[75] F. Xiao, CED: A distance for complex mass functions, IEEE Transactions on Neural Networks and Learning Systems (2020) DOI: 10.1109/TNNLS.2020.2984918.

[76] H. Garg, D. Rani, A robust correlation coefficient measure of complex intuitionistic fuzzy sets and their applications in decision-making, Applied Intelligence 49 (2) (2019) 496-512. 